\title{
Association Between Facility-Level Utilization of Non-pharmacologic Chronic Pain Treatment and Subsequent Initiation of Long-Term Opioid Therapy
}

\author{
Evan P. Carey, $\mathrm{MS}^{7}$, Charlotte Nolan, MPA ${ }^{7}$, Robert D. Kerns, PhD ${ }^{2,3}$, P. Michael Ho, MD, PhD ${ }^{1,4}$, and \\ Joseph W. Frank, MD, MPH ${ }^{1,5}$
}

\begin{abstract}
'Center of Innovation for Veteran-Centered and Value-Driven Care, VA Eastern Colorado Health Care System, Denver, CO, USA; ${ }^{2}$ Pain Research, Informatics, Multimorbidities and Education (PRIME) Center of Innovation, VA Connecticut Healthcare System, West Haven, CT, USA; ${ }^{3}$ Departments of Psychiatry, Neurology and Psychology, Yale University, New Haven, CT, USA; ${ }^{4}$ Division of Cardiology, University of Colorado School of Medicine, Aurora, CO, USA; ${ }^{5}$ Division of General Internal Medicine, University of Colorado School of Medicine, Aurora, CO, USA.
\end{abstract}

BACKGROUND: Expert guidelines recommend nonpharmacologic treatments and non-opioid medications for chronic pain and recommend against initiating longterm opioid therapy (LTOT).

OBJECTIVE: We examined whether veterans with incident chronic pain receiving care at facilities with greater utilization of non-pharmacologic treatments and nonopioid medications are less likely to initiate LTOT.

DESIGN: Retrospective cohort study

PARTICIPANTS: Veterans receiving primary care from a Veterans Health Administration facility with incident chronic pain between 1/1/2010 and 12/31/2015 based on either of 2 criteria: (1) persistent moderate-to-severe patient-reported pain and (2) diagnoses "likely to represent" chronic pain.

MAIN MEASURES: The independent variable was facilitylevel utilization of pain-related treatment modalities (nonpharmacologic, non-opioid medications, LTOT) in the prior calendar year. The dependent variable was patient-level initiation of LTOT ( $\geq 90$ days within 365 days) in the subsequent year, adjusting for patient characteristics.

KEY RESULTS: Among 1,094,569 veterans with incident chronic pain from 2010 to 2015 , there was wide facilitylevel variation in utilization of 10 pain-related treatment modalities, including initiation of LTOT (median, 16\%; range, 5-32\%). Veterans receiving care at facilities with greater utilization of non-pharmacologic treatments were less likely to initiate LTOT in the year following incident chronic pain. Conversely, veterans receiving care at facilities with greater non-opioid and opioid medication utilization were more likely to initiate LTOT; this association was strongest for past year facility-level LTOT initiation (adjusted rate ratio, 2.10; 95\% confidence interval, 2.062.15 , top vs. bottom quartile of facility-level LTOT initiation in prior calendar year).

CONCLUSIONS: Facility-level utilization patterns of nonpharmacologic, non-opioid, and opioid treatments for chronic pain are associated with subsequent patientlevel initiation of LTOT among veterans with incident chronic pain. Further studies should seek to understand

Electronic supplementary material The online version of this article (https://doi.org/10.1007/s11606-018-4324-y) contains supplementary material. which is available to authorized users.

Published online April 9, 2018 facility-level variation in chronic pain care and to identify facility-level utilization patterns that are associated with improved patient outcomes.

KEY WORDS: chronic pain; practice variation; health care delivery; health services research.

J Gen Intern Med 33(Suppl 1):S38-S45

DOI: $10.1007 / \mathrm{s} 11606-018-4324-\mathrm{y}$

(C) Society of General Internal Medicine (outside the USA) 2018

hronic pain is common, disabling, and disproportionately $\smile$ impacts veterans. An estimated $66 \%$ of veterans report any pain and $9 \%$ report severe pain. ${ }^{1}$ Chronic pain is a complex condition involving biological, psychological, and social factors. To address this complexity, expert guidelines endorse multimodal care tailored to the unique needs of each patient. ${ }^{2-}$

${ }^{4}$ Common treatment modalities include medications, procedural treatments, surgery, and non-pharmacologic therapies such as psychological, exercise/movement, and complementary and integrative health approaches. The 2017 Departments of Veterans Affairs and Defense guidelines on opioid therapy for chronic pain strongly recommend non-pharmacologic treatments and non-opioid medications for the treatment of chronic pain. These guidelines also recommend against initiation of long-term opioid therapy (LTOT) for chronic pain, citing the lack of high-quality evidence of benefit and growing evidence of harms. ${ }^{2}$ Utilization of non-pharmacologic treatments and non-opioid medications may improve pain and function and thereby decrease the role of opioid medications. ${ }^{5}$ However, an "opioid-sparing" effect of multimodal and nonpharmacologic interventions has not been demonstrated in prior studies. ${ }^{6}$

Though there is consensus around the importance of a multimodal approach to chronic pain, there remain significant barriers to accessing guideline-concordant pain care for many patients. ${ }^{7}$ These barriers may include inadequate training in chronic pain management among primary care providers, poor 
availability of services such as physical therapy or pain specialty care, and out-of-pocket cost. These barriers may differ across healthcare facilities, healthcare systems, and communities. While wide variation in opioid prescribing has been previously described, little is known about variation in utilization of non-pharmacologic treatments and non-opioid medications for chronic pain. ${ }^{8,9}$ Additionally, at the level of the healthcare facility, it is not known whether variation in utilization of non-pharmacologic treatments and non-opioid medications is associated with patient outcomes.

To address these gaps, we first sought to describe facilitylevel variation in pain-related treatment among a national cohort of veterans with incident chronic pain. We then examined the association between facility-level non-pharmacologic and non-opioid treatment utilization and subsequent patient initiation of LTOT. We hypothesized that veterans receiving care at facilities with greater utilization of non-pharmacologic treatments and non-opioid medications in the prior year would be less likely to initiate LTOT.

\section{METHODS}

\section{Study Population and Data Source}

To identify a cohort of patients with incident chronic pain, we adapted a published algorithm to identify patients with chronic pain using clinical data from the electronic health record. ${ }^{10} \mathrm{We}$ defined chronic pain based on either of two criteria: (1) persistent moderate-to-severe patient-reported pain and (2) the presence of diagnostic codes "likely to represent chronic pain." We defined persistent moderate-to-severe patientreported pain as at least 3 pain numeric rating scores of $\geq 4$ (on a scale from 0 to 10 ) within a single year with at least 30 days between scores. Second, we identified encounters with the International Classification of Diseases, 9th Revision (ICD-9) diagnosis codes "likely to represent chronic pain." 10 Individuals with two or more outpatient encounters within a 2year period or a single inpatient encounter with a chronic pain diagnosis met criteria for chronic pain. We mapped ICD-9 codes to ICD-10 codes using the General Equivalence Mappings tool created by Centers for Medicare and Medicaid Services. ${ }^{11}$ The chronic pain index date was defined as the earliest date on which an individual met either criteria.

To identify veterans with incident chronic pain from 2010 to 2015, we excluded individuals who met chronic pain criteria during the 2-year period from 1/1/2008-12/31/2009.To identify veterans receiving primary care in the Veterans Health Administration (VHA), we excluded individuals with no outpatient primary care visits in the year prior to and including their index date (Fig. 1). We excluded those with any palliative care visits in the prior year or whose date of death was within 1 day of their index date. We excluded veterans if their home facility was not a medical center (e.g., a community-based clinic or nursing home) as access to referral-based pain care resources within VA likely varies widely across facility types.
We excluded veterans if their facility was linked to fewer than 50 veterans as these facilities may produce unstable estimates of facility-level utilization. Finally, individuals with missing demographic data were excluded.

We used clinical and administrative data from the VHA Central Data Warehouse (CDW) from 1/1/2008-12/31/2016, encompassing 2 years before and 1 year after the chronic pain index date for all cohort members (Fig. 2). We obtained demographic data, clinical data, and dates of death from CDW outpatient files. To ascertain medication information, we used the CDW outpatient pharmacy files.

\section{Treatment Utilization}

We adapted prior work on adherence to clinical practice guidelines for opioid therapy for chronic pain to define 10 pain-related treatment modalities. ${ }^{12}$ These modalities included four non-pharmacologic modalities (physical therapy/ occupational therapy [PT/OT], psychosocial treatments, specialty pain clinic, complementary and integrative health), five non-opioid medication classes (non-steroidal antiinflammatory drugs, antidepressants, anticonvulsants, skeletal muscle relaxants, topical analgesics, and opioid medications), and long-term opioid therapy, defined as receipt of $\geq 90$-day supply of an opioid medication within a 365 -day time period. ${ }^{13,14}$ Full definitions of each modality are available in the Online Appendix. We followed each cohort member for up to 1 year after their chronic pain index date to identify whether they utilized each of the 10 treatment modalities noted above (Fig. 2). Individuals were censored during follow-up for mortality or if they utilized palliative care.

\section{Facility-Level Variation}

To make inference on facility-level treatment patterns, we assigned every cohort member to the VHA facility where they were observed to receive most of their primary care. For each individual, we identified outpatient primary care visits occurring within 1 year before and after their chronic pain index date. The home facility was assigned to be the facility where the most primary care visits occurred.

To generate a measure of facility-level variation for each treatment modality, we fit a separate quasi-Poisson general linear model to estimate the expected utilization rate for each modality, adjusted for calendar year of index date, age at index date, gender, race/ethnicity, rurality of patient residence, pain diagnoses, mental health diagnoses, substance use disorder diagnoses, and Quan-Charlson's comorbidity index. In order to estimate the utilization rate over 365 days, we assigned a log offset corresponding to the length of follow-up time for each cohort member. The maximum follow-up time was 365 days, or shorter if the veteran was censored. To calculate the expected utilization rate for each facility-year combination, we aggregated the expected utilization rates for each veteran by facility and baseline year. We then calculated observed over expected rates by dividing the observed number of events by 


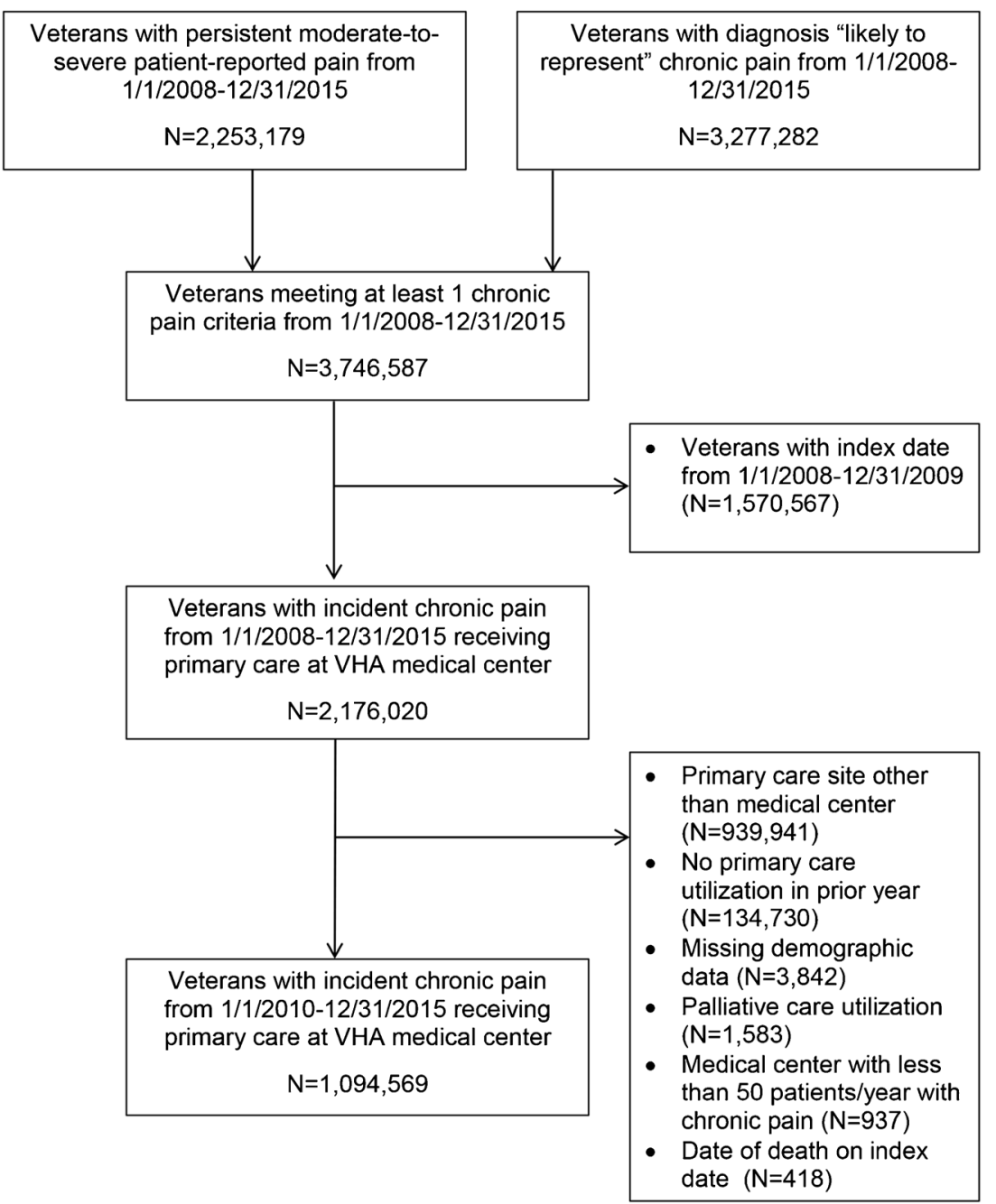

Fig. 1 Cohort flow diagram

a Definition of incident chronic pain
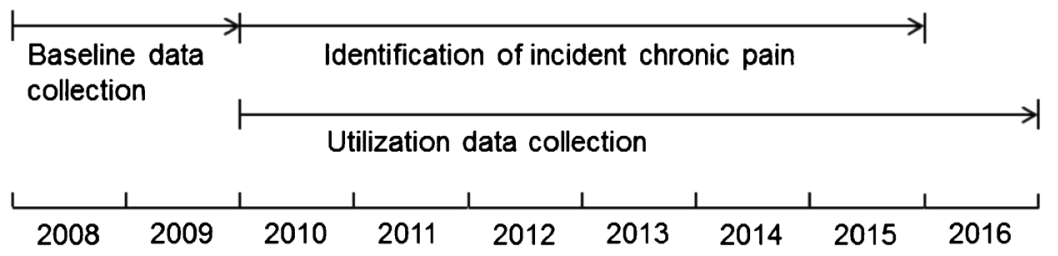

b

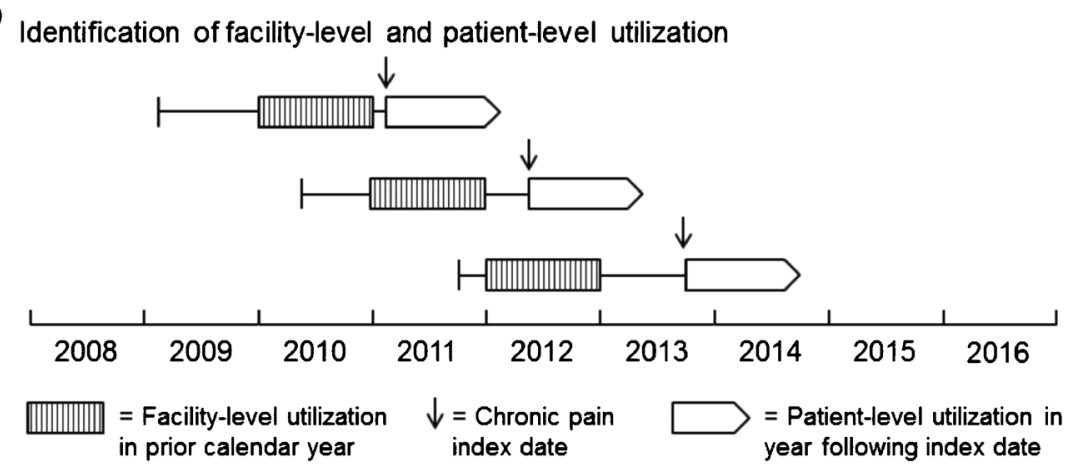

Fig. 2 Definitions of cohort inclusion and subsequent follow-up 
the expected number of events for each facility and year. A value of 1 indicated a facility had exactly the expected (or average) utilization that year. Values less than 1 indicated the facility had less than the expected rate of events, and values greater than 1 indicated the facility had a higher than expected rate of events. These observed over expected ratios were then split into quartiles within each year, which can be interpreted as a measure of facility-level variation in pain treatment modalities by year, adjusted for patient characteristics.

\section{Covariates}

Gender, date of birth, race/ethnicity, and location of residence were obtained from administrative files. Veterans reporting multiple races were coded as reporting each individually. We assessed rurality of individuals' residence using the VHA's Urban/Rural/Highly Rural classification scheme. ${ }^{15}$ We identified pain, mental health, and substance use disorder diagnoses recorded in the 2 years before or 6 months after cohort members' chronic pain index date, categorized based on prior pain studies. ${ }^{14,16,17}$ We implemented the Quan-Charlson's comorbidity index using outpatient and inpatient encounter data in the 2 years prior and 6 months after index date. ${ }^{18}$ Finally, we obtained data on facility rurality and complexity. The VHA maintains a classification of facility traits including facility type, complexity, and urban/rural status. Urban/rural status is defined based on the geographic location of each facility. Facility complexity includes 1a, 1b, 1c, 2, and 3 with 1a being the most complex and 3 being the least complex. ${ }^{19}$

\section{Statistical Analyses}

We first calculated descriptive statistics of the study cohort and unadjusted facility-level variation for each modality. We then examined the association between facility-level pain treatment utilization (categorized as quartiles) in the calendar year prior to an individual's chronic pain index date and subsequent patient-level LTOT initiation. Among cohort members with an index date of $1 / 1 / 2011-12 / 31 / 2015$, we fit quasi-Poisson models to estimate the association between each facility-level treatment modality quartile in the prior calendar year and the rate of patient-level LTOT initiation in the 365 days following chronic pain index date, adjusted for patient and facility characteristics. As prior calendar year facility-level data were not available for individuals with an index date in 2010, these models included individuals with chronic pain index dates from 2011 to 2015 . We fit a separate model for each modality (Fig. 3, Model 1) and then a single model with all modalities (Fig. 3, Model 2). The results of these models were summarized as rate ratios and $95 \%$ confidence intervals. As a sensitivity analysis, we excluded cohort members prescribed LTOT in the 2 years prior to their chronic pain index date and conducted the above analyses among this "opioid naïve" cohort.

The activities undertaken in this project supported VHA operational programs and did not constitute research; institutional review board approval was therefore not required. This work was supported by the Department of Veterans Affairs, Quality Enhancement Research Initiative (QUE 15468). The funding sources had no role in the design and conduct of the study; collection, management, analysis, or interpretation of the data; or preparation, review, or approval of the manuscript.

\section{RESULTS}

From 2010 to 2015, there were 1,094,569 veterans with incident chronic pain receiving primary care in the Veterans Health Administration (Fig. 1). This cohort was predominantly male (90\%) and White (74\%); $46 \%$ were 60 years of age or older (Table 1). Back pain (28\%) and neck or other joint pain (36\%) were the most common pain diagnoses. Baseline comorbidities included depression (19\%), post-traumatic stress disorder (14\%), anxiety (11\%), and alcohol use disorder (9\%).

There was substantial facility-level variation in utilization of pain-related treatment modalities during the first year following incident chronic pain diagnosis (Table 2). Among nonpharmacologic modalities, psychosocial treatments were the most frequently provided modality (median, $42 \%$, interquartile range [IQR], 38-47\%) followed by PT/OT (median, 39\%; IQR, 34-45\%). There was wide facility-level variation in utilization of non-opioid and opioid medications. Across VHA medical centers, initiation of long-term opioid therapy in the year following incident chronic pain ranged from 5 to $32 \%$ (median, 16\%; IQR, 12-19\%).

For 3 of 4 non-pharmacologic pain treatment modalities examined, facility-level utilization in the prior calendar year was associated with decreased patient-level LTOT initiation in the year following individuals' chronic pain index date, after adjustment for patient and facility characteristics (Fig. 3, Model 1). For example, veterans receiving care at medical centers in the top quartile for PT/OT in the prior calendar year had a $22 \%$ decreased rate of initiating LTOT (adjusted rate ratio [ARR] 0.78, 95\% confidence interval [CI] 0.77-0.79) compared to veterans receiving care at medical centers in the bottom quartile. For 4 of 5 non-opioid medication classes examined, facility-level utilization was associated with increased patient-level LTOT initiation in the year following individuals' chronic pain index date, after adjustment for patient characteristics. There was an increased rate of LTOT initiation for veterans receiving care at medical centers in the top quartile for skeletal muscle relaxants (ARR 1.63, 95\% CI 1.60-1.65), anticonvulsants (ARR 1.41, 95\% CI 1.39-1.44), antidepressants (ARR 1.23, 95\% CI 1.21-1.25), and NSAIDs (ARR $1.42,95 \%$ CI 1.40-1.44) compared to veterans receiving care at medical centers in the bottom quartile for each medication class.

These associations were strongest for facility-level LTOT quartile and subsequent LTOT initiation. After adjustment for both patient and facility characteristics and facility-level 


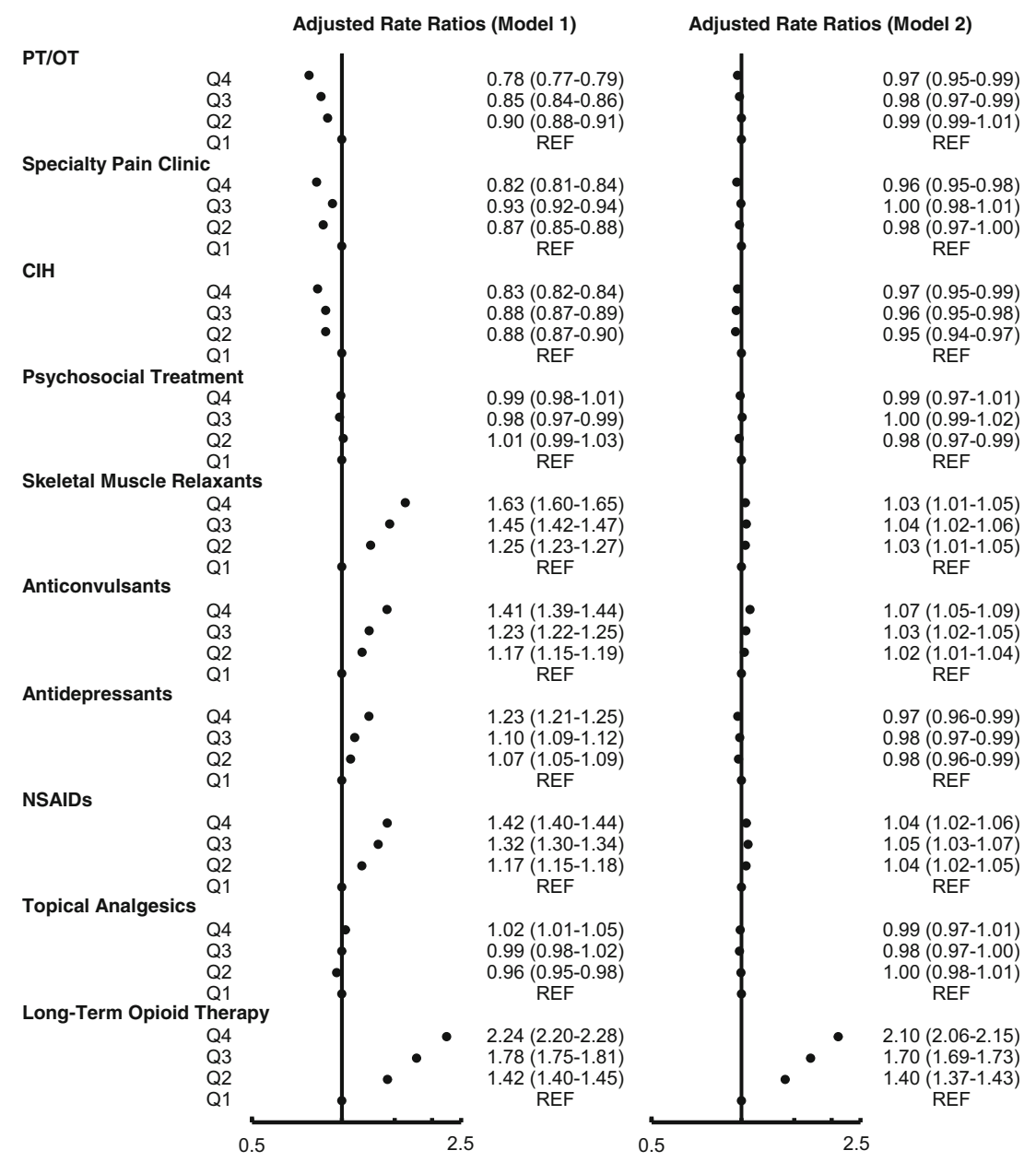

Fig. 3 Rate of long-term opioid therapy initiation according to facility-level quartiles of prior year pain care utilization among veterans with incident chronic pain*

utilization of pain treatment modalities, veterans receiving care at medical centers in the highest quartile for LTOT in the prior calendar year had a $110 \%$ increased rate of initiating LTOT in the year following their chronic pain index date (ARR 2.10, 95\% CI 2.06-2.15) compared to veterans receiving care at medical centers in the lowest quartile (Fig. 3, Model 2 ). In a sensitivity analysis excluding the $5.7 \%$ of cohort members prescribed LTOT in the 2 years prior to their chronic pain index date, model results were similar to those in the primary analyses.

\section{DISCUSSION}

In this study of $1,094,569$ veterans with incident chronic pain receiving care at 176 VHA medication centers from 2010 to 2015 , there was substantial variation in pain treatment utilization among medical centers. Prior studies have documented regional variation in opioid prescribing. ${ }^{8,9}$ In 2015 , prescribed opioid dose varied more than 6-fold between US counties in the top and bottom quartile; in this study, facilities varied by 3 - fold in any opioid utilization (20-58\%) and 6-fold in LTOT (5-32\%). To our knowledge, facility-level variation in nonpharmacologic treatments and non-opioid pain medications has not previously been described in a national healthcare system.

As hypothesized, greater facility-level utilization of nonpharmacologic modalities was associated with decreased LTOT initiation. Facility-level variation in nonpharmacologic pain treatment utilization likely involves facility, provider, and patient factors. For facilities, decisions to prioritize support for non-pharmacologic treatment providers such as physical therapists or psychologists likely play an important role; patients cannot utilize services that are unavailable. For providers, prior training and comfort with nonpharmacologic chronic pain management may differ across facilities ${ }^{20-22}$; local "champions" may drive facility-level change. ${ }^{23,24}$ For patients, past experience with and confidence in the effectiveness of non-pharmacologic modalities may differ across facilities. ${ }^{25,26}$ Several potential mechanisms may underlie the relationship between past year facility-level non-pharmacologic pain treatment utilization and rate of 
Table 1 Characteristics of Veterans with Incident Chronic Pain in the Veterans Health Administration, 2010-2015 $(N=1,094,569)$

\begin{tabular}{ll}
\hline \hline Characteristic & Total \\
\hline Male sex, $N(\%)$ & $982,548(90 \%)$ \\
Race*, $N(\%)$ & \\
White & $759,323(74 \%)$ \\
Black & $235,872(24 \%)$ \\
Other race & $36,816(3 \%)$ \\
Missing & $73,365(7 \%)$ \\
Hispanic ethnicity, $N(\%)$ & $77,762(7 \%)$ \\
Age, $N$ (\%) & \\
$<30$ & $111,444(10 \%)$ \\
$30-60$ & $479,092(44 \%)$ \\
$>60$ & $504,033(46 \%)$ \\
Urban residence, $N(\%)$ & $732,755(67 \%)$ \\
Pain diagnoses, $N$ (\%) & \\
Back pain & $310,405(28 \%)$ \\
Neck/other joint pain & $398,359(36 \%)$ \\
Neuropathic pain & $36,252(3 \%)$ \\
Migraine & $58,225(5 \%)$ \\
Fibromyalgia & $10,072(1 \%)$ \\
Mental health diagnoses, $N(\%)$ & \\
Depression & $208,051(19 \%)$ \\
Post-traumatic stress disorder & $151,548(14 \%)$ \\
Anxiety & $116,159(11 \%)$ \\
Bipolar disorder & $27,604(3 \%)$ \\
Substance use disorder diagnoses, $N(\%)$ & \\
Alcohol use disorder & $98,551(9 \%)$ \\
Opioid use disorder & $17,802(2 \%)$ \\
Other substance use disorder & $62,552(6 \%)$ \\
Charlson's comorbidity index, mean (SD) & $0.63(1.43)$ \\
\hline SD standard deviation & \\
$*$ Race categories not mutually exclusive & \\
&
\end{tabular}

LTOT initiation. First, greater access to and engagement with non-pharmacologic pain treatment modalities may improve

Table 2 Facility-Level Characteristics of 176 Medical Centers in the Veterans Health Administration, 2010-2015 $(N=1,094,569)$

\begin{tabular}{lll}
\hline \hline & Median (IQR) & Range (\%) \\
\hline Male sex & $91 \%(89-93 \%)$ & $55-96$ \\
Race/ethnicity & $84 \%(72-93 \%)$ & $25-98$ \\
White & $13 \%(5-25 \%)$ & $0-74$ \\
Black & $1 \%(1-3 \%)$ & $0-37$ \\
Other race & $5 \%(3-8 \%)$ & $0-28$ \\
Missing & $2 \%(1-7 \%)$ & $0-93$ \\
Hispanic ethnicity & $10 \%(8-11 \%)$ & $5-19$ \\
Age & $41 \%(37-46 \%)$ & $23-65$ \\
<30 & $49 \%(43-54 \%)$ & $18-71$ \\
$30-60$ & $69 \%(47-87 \%)$ & $5-99$ \\
S60 & & \\
Urban residence & $42 \%(38-47 \%)$ & $22-92$ \\
Pain treatment utilization* & $39 \%(34-45 \%)$ & $16-61$ \\
Non-pharmacologic modalities & $9 \%(5-14 \%)$ & $1-48$ \\
Psychosocial treatment & $2 \%(1-5 \%)$ & $0-20$ \\
PT/OT & & \\
Specialty pain clinic & $19 \%(16-23 \%)$ & $9-33$ \\
CIH & $13 \%(12-15 \%)$ & $6-24$ \\
Non-opioid medications & $7 \%(6-9 \%)$ & $3-13$ \\
Skeletal muscle relaxants & $42 \%(37-47 \%)$ & $25-57$ \\
Anticonvulsant medications & $9 \%(7-14 \%)$ & $1-30$ \\
Antidepressant medications & & \\
NSAIDs & & $21-57$ \\
Topical medications & $39 \%(35-43 \%)$ & $5-32$ \\
Opioid medications & $16 \%(12-19 \%)$ \\
Any opioid medication & Long-term opioid therapy & \\
\hline
\end{tabular}

CIH complementary and integrative health, NSAID non-steroidal antiinflammatory drug, PT/OT physical therapy and occupational therapy *Treatment utilization denotes any clinical encounter or prescription within 1 year of chronic pain index date among veterans with incident chronic pain from 2010 to 2015 important patient outcomes such as pain and function, decreasing the need for LTOT. Alternatively, more judicious prescribing of opioids may lead to both greater concurrent utilization of non-pharmacologic treatment options and continued judicious opioid prescribing in the subsequent year.

Unexpectedly, greater facility-level utilization of several non-opioid medication classes was associated with increased LTOT initiation. This association may reflect differences in the "culture" of pain management across facilities. Providers' level of familiarity and comfort with medications may encompass both opioids and non-opioids alike, leading analgesic medications across multiple classes to be relatively "available" in some facilities. Similarly, patients' expectations around the role of medications, both opioids and non-opioids, may also differ across facilities and communities.

Finally, facility-level utilization of LTOT in the prior year was most strongly associated with subsequent initiation of LTOT among veterans with incident chronic pain. At facilities in the highest quartile for LTOT in the prior year, veterans with incident chronic pain had a $110 \%$ increased rate of initiating LTOT in the subsequent year compared to those receiving care at medical centers in the lowest quartile; this rate increased in a stepwise fashion across quartiles. Previous studies have examined patient, provider, and facility factors that increase risk of LTOT. Patient-level predictors of LTOT initiation have included male sex, greater pain intensity, nicotine dependence, mental health, and substance use disorder diagnoses. ${ }^{13,14,27,28}$ Physicians' opioid prescribing practices at hospital discharge and in the emergency department have been shown to impact risk of long-term opioid use. ${ }^{29,30}$ Prior studies of facility-level interventions have primarily focused on strategies to manage opioid-related risks. ${ }^{31,32}$ As with the relationship seen with non-opioid medications, this finding may also represent a "culture" at the facility level around the appropriate role of opioid medications for chronic pain. Alternatively, variation may be driven by outliers within facilities where a small number of providers are substantially more or less likely to prescribe opioid compared to their peers.

Study findings should be interpreted in the context of the study's limitations. First, this is an observational study; the associations identified should not be interpreted as causal. Second, we are not able to characterize the indication or appropriateness of individual treatment modalities for individual patients. For example, psychosocial treatment for a veteran with chronic pain may have targeted a co-occurring condition such as depression. In a biopsychosocial model of chronic pain, treatment of co-occurring conditions can be considered a component of multimodal pain care. Third, we examined a veteran population and our results may not be generalizable to other populations or healthcare systems. Finally, we are not able to identify pain treatment received in other healthcare systems, in the community, or as self-management. We excluded community-based clinics from this analysis as availability of non-pharmacologic pain treatments likely differs in these settings compared to medical centers. 
These limitations highlight important next steps for researchers and policymakers. Randomized trials are needed to determine the effectiveness of non-pharmacologic pain treatment modalities to prevent or decrease long-term opioid use while effectively managing chronic pain. These data could assist patients and providers in selecting the nonpharmacologic strategies that are most likely to provide meaningful benefit. As expert consensus recommends non-opioid treatment of chronic pain, evidence-based models of care are needed to facilitate access to the range of chronic pain treatments. Future studies should seek to inform implementation of the range of non-pharmacologic and non-opioid treatment options across diverse, real-world clinical settings. These efforts will require support from healthcare leaders and significant investment in the health system's capacity to deliver the range of evidence-based non-pharmacologic treatments. ${ }^{33}$ The return on these investments should be measured in gains in function and improvements in quality of life but may also include prevention of opioid-related harms.

In conclusion, among a national cohort of veterans with incident chronic pain, we identified substantial facility-level variation for each of 4 non-pharmacologic pain treatments and 6 medication classes. This facility-level variation was associated with subsequent patient-level rate of LTOT initiation. Further studies should seek to understand facility-level variation in chronic pain care and to identify facility-level utilization patterns that are associated with improved patient outcomes.

Corresponding Author: Joseph W. Frank, MD, MPH; Division of General Internal Medicine, University of Colorado School of Medicine, Aurora, CO, USA (e-mail: joseph.frank@ucdenver.edu).

\begin{abstract}
Authors' contribution All authors made substantial contributions to conception and design, acquisition of data, or analysis and interpretation of data; drafting the article or revising it critically for important intellectual content; and final approval of the version to be published.
\end{abstract}

Funding This work was supported by the Department of Veterans Affairs, Quality Enhancement Research Initiative (QUE 15-468). Dr. Frank was supported in part by VA Health Services Research \& Development Career Development Award (HSR\&D CDA 15-059). Dr. Kerns was supported in part by a VA Health Services Research \& Development Center of Innovation (CIN 13-047). The funding sources had no role in the design and conduct of the study; collection, management, analysis, or interpretation of the data; or preparation, review, or approval of the manuscript. The views expressed in this article are those of the authors and do not necessarily reflect the position or policy of the Department of Veterans Affairs or the US Government.

\section{Compliance with ethical standards:}

Prior presentations: This manuscript has not been previously published and is not under consideration in the same or substantially similar form in any other peer-reviewed media. This work will be presented as a poster at the upcoming VA HSR\&D/QUERI meeting on July 18-20, 2017, in Washington, DC.

Conflict of interest: The authors declare that they do not have a conflict of interest.

\section{Abbreviations}

$\mathrm{CIH} \quad$ comlementary and integrative health

PT/OT physical therapy/occupational therapy

NSAIDs non-steroidal anti-inflammatory drugs

* $\quad$ Sample size across all years, N =858,199

$\dagger \quad$ Adjusted rate ratios and $95 \%$ confidence intervals for initiation of long-term opioid therapy during year following chronic pain index date. Adjusted estimates generated using a quasi-Poisson general linear model. Model 1 adjusted for patient age at index date, gender, race/ethnicity, calendar year of index date, rurality of patient residence, pain diagnoses (back pain, neck, and other joint pain), mental health diagnoses (depression, anxiety, post-traumatic stress disorder, bipolar disorder), substance use disorder diagnoses (alcohol use disorder, opioid use disorder, other substance use disorder), Charlson's comorbidity index, facility rurality, and facility complexity.

$\S \quad$ Model 2 adjusted for all Model 1 variables plus each of 10 facility-level pain treatment modality variables, categorized as quartiles.

\section{REFERENCES}

1. Nahin RL. Severe Pain in Veterans: The Effect of Age and Sex, and Comparisons With the General Population. J Pain 2017;18:247-54.

2. VA/DoD Clinical Practice Guideline for Opioid Therapy for Chronic Pain: Department of Veterans Affairs and Department of Defense; 2017.

3. Gaseem A, Wilt TJ, McLean RM, Forciea MA, Clinical Guidelines Committee of the American College of P. Noninvasive Treatments for Acute, Subacute, and Chronic Low Back Pain: A Clinical Practice Guideline From the American College of Physicians. Ann Intern Med 2017;166:514-30.

4. Dowell D, Haegerich TM, Chou R. CDC Guideline for Prescribing Opioids for Chronic Pain - United States, 2016. MMWR Recomm Rep 2016;65:1-49.

5. Peterson $\mathbf{K}$, Anderson J, Bourne D, Mackey $\mathbf{K}$, Helfand M. Evidence Brief: Effectiveness of Models Used to Deliver Multimodal Care for Chronic Musculoskeletal Pain. VA Evidence-based Synthesis Program, Department of Veterans Affairs Health Services Research \& Development Service; 2017.

6. Peterson K, Anderson J, Ferguson L, Mackey K. Evidence Brief: The Comparative Effectiveness of Selected Complementary and Integrative Health (CIH) Interventions for Preventing or Reducing Opioid Use in Adults with Chronic Neck, Low Back, and Large Joint Pain. VA Evidencebased Synthesis Program, Department of Veterans Affairs Health Services Research \& Development Service; 2016.

7. Relieving Pain in America: A Blueprint for Transforming Prevention, Care, Education, and Research. Washington, DC: Institute of Medicine; 2011.

8. Mosher HJ, Krebs EE, Carrel M, Kaboli PJ, Weg MW, Lund BC. Trends in prevalent and incident opioid receipt: an observational study in Veterans Health Administration 2004-2012. J Gen Intern Med 2015;30:597-604.

9. Guy G, Zhang K, Bohm M, et al. Vital Signs: Changes in Opioid Prescribing in the United States, 2006-2015: MMWR Morb Mortal Wkly Rep. 2017;66(26). https://www.cdc.gov/mmwr/volumes/66/wr/ mm6626a4.htm?s_cid=mm6626a4_w. Accessed July 10, 2017.

10. Tian TY, Zlateva I, Anderson DR. Using electronic health records data to identify patients with chronic pain in a primary care setting. J Am Med Inform Assoc 2013;20:e275-80.

11. ICD-10-CM/PCS Resources: Tools. Healthcare Cost and Utilization Project (HCUP). Agency for Healthcare Research and Quality, 2016. www.hcup-us.ahrq.gov/datainnovations/icd10_tools.jsp. Accessed July 12,2017

12. Midboe AM, Lewis ET, Paik MC, et al. Measurement of adherence to clinical practice guidelines for opioid therapy for chronic pain. Transl Behav Med 2012;2:57-64.

13. Edlund MJ, Martin BC, Devries A, Fan MY, Braden JB, Sullivan MD. Trends in use of opioids for chronic noncancer pain among individuals 
with mental health and substance use disorders: the TROUP study. Clin J Pain 2010;26:1-8.

14. Dobscha SK, Morasco BJ, Duckart JP, Macey T, Deyo RA. Correlates of prescription opioid initiation and long-term opioid use in veterans with persistent pain. Clin J Pain 2013;29:102-8.

15. West AN, Lee RE, Shambaugh-Miller MD, et al. Defining "rural" for veterans' health care planning. J Rural Health 2010;26:301-9.

16. Goulet JL, Kerns RD, Bair M, et al. The musculoskeletal diagnosis cohort: examining pain and pain care among veterans. Pain 2016;157:1696-703.

17. Frank JW, Carey EP, Fagan KM, et al. Evaluation of a telementoring intervention for pain management in the Veterans Health Administration. Pain Med 2015; 16:1090-100.

18. Guan H, Li B, Couris CM, et al. Updating and validating the Charlson comorbidity index and score for risk adjustment in hospital discharge abstracts using data from 6 countries. Am J Epidemiol 2011;173:676-82.

19. Facility Complexity Levels. VHA Office of Productivity, Efficiency \& Staffing (OPES). http://opes.vssc.med.va.gov/FacilityComplexityLevels/ Pages/default.aspx. Accessed Nov. 7, 2017.

20. Berg KM, Arnsten JH, Sacajiu G, Karasz A. Providers' experiences treating chronic pain among opioid-dependent drug users. J Gen Intern Med 2009:24:482-8.

21. Dobscha SK, Corson K, Flores JA, Tansill EC, Gerrity MS. Veterans affairs primary care clinicians' attitudes toward chronic pain and correlates of opioid prescribing rates. Pain Med 2008;9:564-71.

22. Upshur CC, Luckmann RS, Savageau JA. Primary care provider concerns about management of chronic pain in community clinic populations. J Gen Intern Med 2006;21:652-5.

23. Dorflinger L, Moore B, Goulet J, et al. A partnered approach to opioid management, guideline concordant care and the stepped care model of pain management. J Gen Intern Med 2014;29 Suppl 4:870-6.
24. Williams KM, Kirsh S, Aron D, et al. Evaluation of the Veterans Health Administration's Specialty Care Transformational Initiatives to Promote Patient-Centered Delivery of Specialty Care: A Mixed-Methods Approach. Telemed J E Health 2017;23:577-89.

25. Simmonds MJ, Finley EP, Vale S, Pugh MJ, Turner BJ. A qualitative study of veterans on long-term opioid analgesics: barriers and facilitators to multimodality pain management. Pain Med 2015;16:726-32.

26. Park J, Hirz CE, Manotas K, Hooyman N. Nonpharmacological pain management by ethnically diverse older adults with chronic pain: barriers and facilitators. J Gerontol Soc Work 2013;56:487-508.

27. Edlund MJ, Austen MA, Sullivan MD, et al. Patterns of opioid use for chronic noncancer pain in the Veterans Health Administration from 2009 to 2011. Pain 2014;155:2337-43.

28. Krebs EE, Lurie JD, Fanciullo G, et al. Predictors of long-term opioid use among patients with painful lumbar spine conditions. J Pain 2010;11:44-52.

29. Calcaterra SL, Yamashita TE, Min SJ, Keniston A, Frank JW, Binswanger IA. Opioid Prescribing at Hospital Discharge Contributes to Chronic Opioid Use. J Gen Intern Med 2016;31:478-85.

30. Barnett ML, Olenski AR, Jena AB. Opioid-Prescribing Patterns of Emergency Physicians and Risk of Long-Term Use. N Engl J Med 2017;376:663-73.

31. Coffin PO, Behar E, Rowe C, et al. Nonrandomized Intervention Study of Naloxone Coprescription for Primary Care Patients Receiving Long-Term Opioid Therapy for Pain. Ann Intern Med 2016;165:245-52.

32. Turner JA, Saunders K, Shortreed SM, et al. Chronic opioid therapy risk reduction initiative: impact on urine drug testing rates and results. J Gen Intern Med 2014;29:305-11.

33. Gellad WF, Good CB, Shulkin DJ. Addressing the Opioid Epidemic in the United States: Lessons From the Department of Veterans Affairs. JAMA Intern Med 2017;177(5):611-612. 